\title{
Evaluation of microvascular changes in the
} macular area of eyes with rhegmatogenous retinal detachment without macular involvement using swept-source optical coherence tomography angiography

This article was published in the following Dove Press journal: Clinical Ophthalmology

\section{Yuji Yoshikawa \\ Takuhei Shoji \\ Junji Kanno \\ Hisashi Ibuki \\ Kimitake Ozaki \\ Hirokazu Ishii \\ Yoshikazu Ichikawa \\ Itaru Kimura \\ Kei Shinoda \\ Department of Ophthalmology, Saitama Medical University, Saitama, Japan}

Correspondence: Kei Shinoda Department of Ophthalmology, Saitama Medical University, 38 Morohongo Moroyama-machi, Iruma, Saitama 350-0495, Japan

Tel $+8 \mid 49276 \quad 250$

Fax +8I 492958002

Email shinok@saitama-med.ac.jp
Purpose: To evaluate microvascular changes in the macular area of eyes with rhegmatogenous retinal detachment (RRD) without macular involvement (macula-on RRD) using swept-source optical coherence tomography angiography (SS-OCTA).

Patients and methods: Five patients with macula-on RRD were eligible for analysis. All patients underwent SS-OCTA examination (Triton) for the macular area. The healthy fellow eyes were included as controls. The vessel density (VD) was calculated using binarization, and the foveal avascular zone (FAZ) was measured. The VD and FAZ area were compared between the eyes with RRD and the fellow eyes using the Wilcoxon signed rank test.

Results: The patients' clinical characteristics were as follows: age, 49.0 years $(21.0,54.0)$ (median $[25,75$ th percentile]); preoperative best-corrected visual acuity, $-0.08(-0.08,0.11)$ for RRD and $-0.08(-0.08,-0.03)$ for the fellow eye $(P=0.50)$; and axial length, $27.0(25.1,28.7) \mathrm{mm}$ for RRD and $27.4(25.6,28.5) \mathrm{mm}$ for the fellow eye $(P=0.31)$. The parafoveal VD was not significantly different between the eyes with RRD and the fellow eyes $(P=1.00$ for the superficial retina and $P=0.44$ for the whole retina). The FAZ area was also similar for the eyes with RRD and the fellow eyes ( $P=0.31$ for the superficial retina and $P=0.13$ for the whole retina).

Conclusion: The findings of this study suggest that the macular microvasculature remains intact in eyes with macula-on RRD.

Keywords: rhegmatogenous retinal detachment, macula, optical coherence tomography angiography, vessel density

\section{Introduction}

Rhegmatogenous retinal detachment (RRD) is characterized by separation of the sensory retina from the retinal pigment epithelium and is caused by a retinal tear. This condition will lead to the loss of vision in the absence of treatment. RRD is commonly treated with scleral buckling or pars plana vitrectomy with air, gas, or silicone oil used for tamponade in selected cases. ${ }^{1,2}$ Although primary surgical success rates range from $80 \%$ to $92 \%, 1,3,4$ anatomical success does not necessarily lead to good visual outcomes. Maintenance of the foveal structure is related to the postoperative visual outcome..$^{5-8}$

Previous reports showed a relationship between RRD and vascular changes in the macular region. Ito et $\mathrm{al}^{9}$ described a reduction in the vascular flow in the central retinal artery and the short posterior ciliary artery after scleral buckling, whereas Iwase et $\mathrm{al}^{10}$ 
reported a decrease in the retinal blood flow in the optic nerve head using laser speckle flowgraphy for eyes with RRD, which successfully recovered after RRD repair with vitrectomy.

Recently, optical coherence tomography angiography (OCTA) has become clinically available. OCTA is a new, noninvasive method for visualizing the ocular microvasculature. Compared with fluorescein angiography, OCTA can detect changes in the microcirculation without dye leakage. Furthermore, OCTA can perform layer-by-layer analysis and provide information for the deeper vascular areas. ${ }^{11}$ Hwang et al ${ }^{12}$ reported that OCTA allows better detection of capillary dropout and early retinal neovascularization, without dye leakage, than does fluorescein angiography in patients with diabetic retinopathy.

Thus, the purpose of the present study was to evaluate the macular microvasculature with regard to the vessel density (VD) and foveal avascular zone (FAZ) area in eyes with RRD without macular involvement (macula-on RRD) and compare the findings with those for the fellow eyes using swept-source OCTA (SS-OCTA).

\section{Materials and methods Study population}

This case series was approved by the ethics committee of Saitama Medical University and was conducted in accordance with the tenets of the Declaration of Helsinki. Japanese participants with macula-on RRD were recruited from the ophthalmology outpatient clinic at Saitama Medical University (Saitama, Japan). Patients were included if they were 18 years of age or older, fulfilled the eligibility requirements detailed below, and signed an informed consent form between April 2017 and October 2017.
All participants underwent a comprehensive ophthalmic examination, including slit-lamp biomicroscopy, intraocular pressure measurement (noncontact tonometry; Tonoref II; Nidek Co., Ltd., Aichi, Japan), fundus photography (CX-1, Canon Inc., Tokyo, Japan), axial length measurement (Optical Biometer OA-2000; Tomey Corp., Aichi, Japan), and SS-OCTA (Triton, Topcon Co., Tokyo Japan).

The exclusion criteria were as follows: RRD with parafoveal involvement and poor image quality.

\section{Optical coherence tomography angiography}

A $3 \times 3 \mathrm{~mm}$ OCTA image centered on the fovea was acquired using an SS-OCT device (Triton, Topcon) featuring a central wavelength of 1,050 nm, a speed of 100,000 A-scans per second, and an axial and a transverse tissue resolution of 8 and $20 \mu \mathrm{m}$, respectively. OCT-A software was used for the acquisition of $3 \times 3 \mathrm{~mm}$ cubes, with each cube composed of $320 \times 320$ pixels. The angiography images were processed using OCT Angiography Ratio Analysis, which is a motion contrast measure based on the ration method (Figure 1). The SS-OCTA software generates en face images from slabs at different layers using an automated segmentation mode (Superficial and Full Macula modes). Each segmentation is defined as follows.

\section{Superficial mode}

The inner boundary is $2.6 \mu \mathrm{m}$ below the internal limiting membrane, and the outer boundary is $15.6 \mu \mathrm{m}$ below the inner plexiform layer. This area is referred to as the superficial retina in the present study.
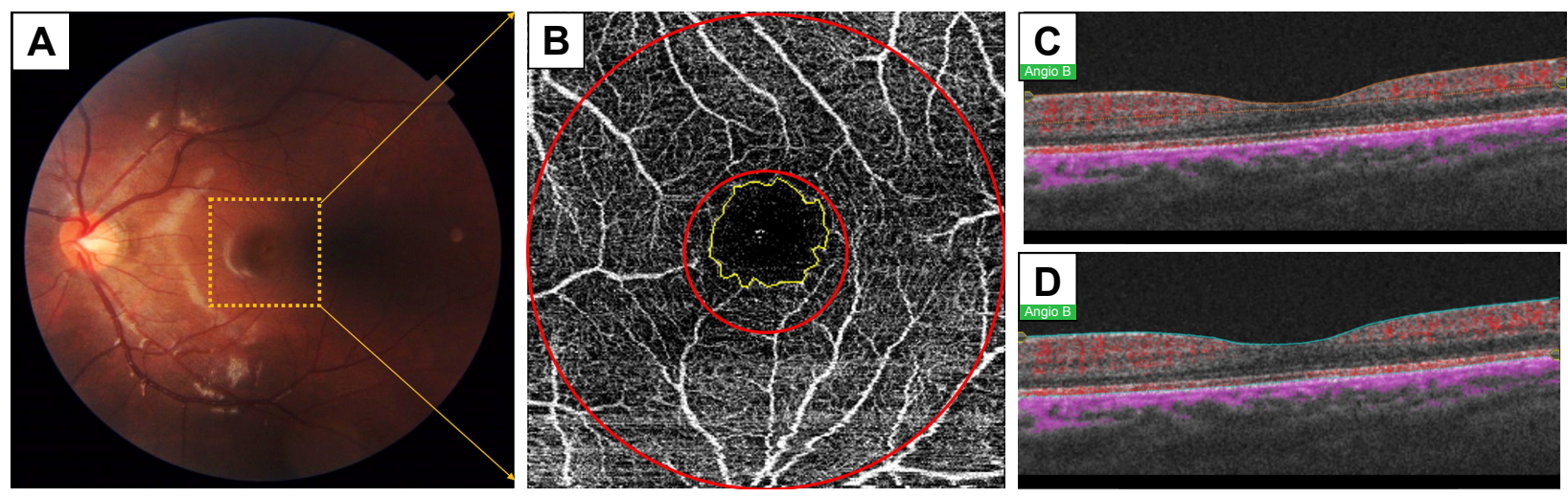

Figure I Methods of VD and FAZ measurement using SS-OCTA for eyes with macula-on RRD.

Notes: A $3 \times 3 \mathrm{~mm}$ SS-OCTA en face image has been obtained for the macular area (yellow dot, A), and the parafoveal area (area enclosed by two concentric circles) and FAZ (yellow line, B) are measured. SS-OCTA en face images have been obtained with the superficial mode (C) and full macular mode (D).

Abbreviations: VD, vessel density; FAZ, foveal avascular zone; SS-OCTA, swept-source optical coherence tomography angiography; RRD, rhegmatogenous retinal detachment. 


\section{Full macula mode}

The inner boundary is internal limiting membrane and the outer boundary is retinal pigment epithelium. This area is referred to as the whole retina in the present study (Figure 1).

\section{Evaluation of microvascular signal and FAZ area}

For macular scans, the parafoveal region was measured in a circle with an outer diameter of $3 \mathrm{~mm}$ and an inner diameter of $1 \mathrm{~mm}$ (Figure 1). The VD was calculated in the parafoveal region for the superficial retina and whole retina. To analyze the angiography signals, we performed Phansalkar analysis for binarization of the OCTA images using ImageJ software (developed by Wayne Rasband, National Institutes of Health; available at $\mathrm{http}$ ://rsb.info.nih.gov/ij/index.html) for obtaining the microvascular signals. ${ }^{13,14}$ We calculated the VD (\%) as a proportion of the angiography signal relative to that in each region (Figures 1 and 2).

For measurement of the FAZ area, all edge points were manually connected to each other along the border
Case 1 L) RRD
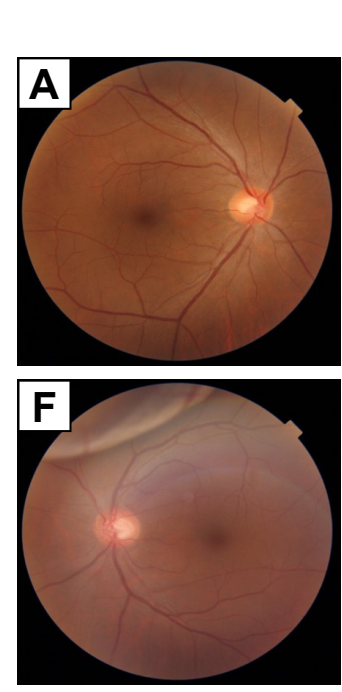

Case 2 L) RRD
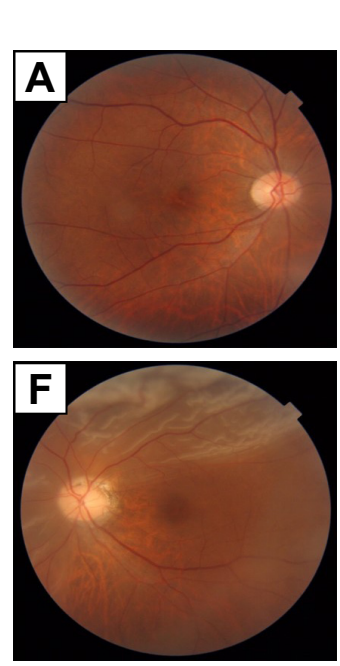

Superficial retina
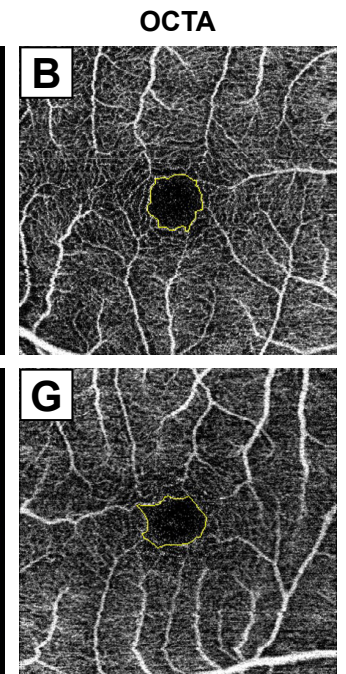

Binarization
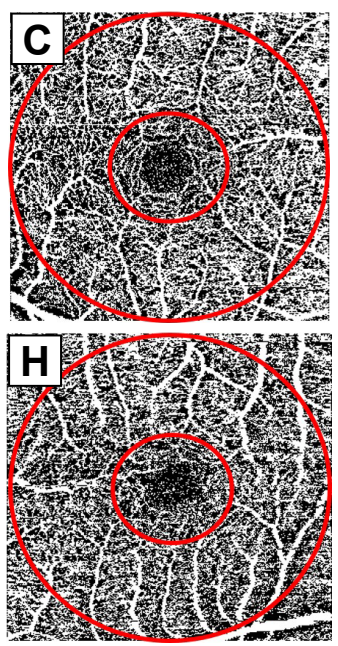

\section{Whole retina}

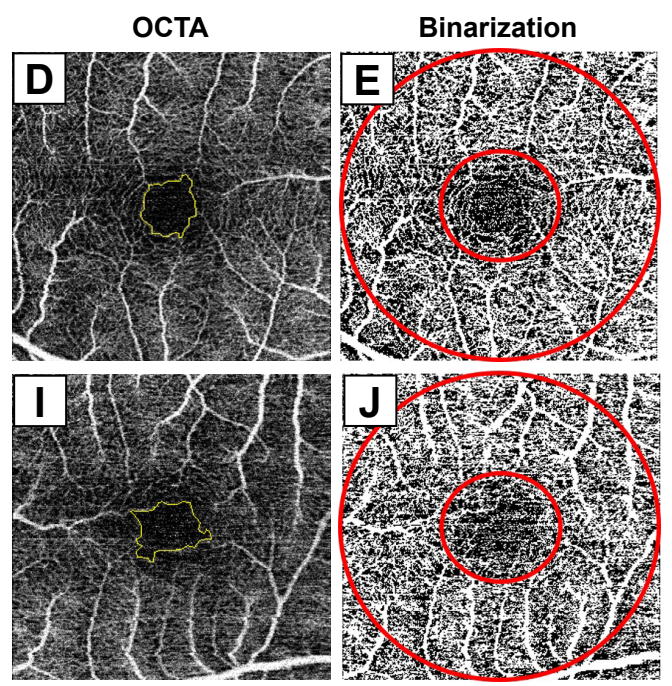

Superficial retina
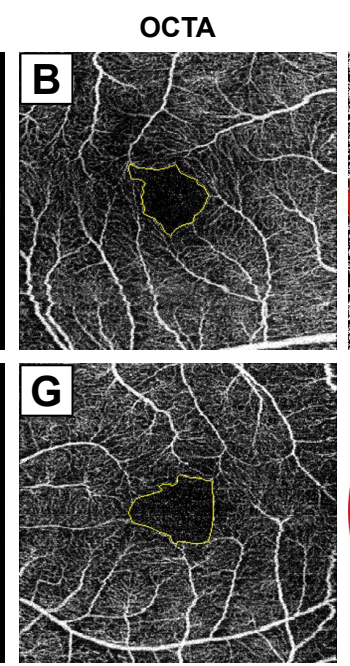

Binarization
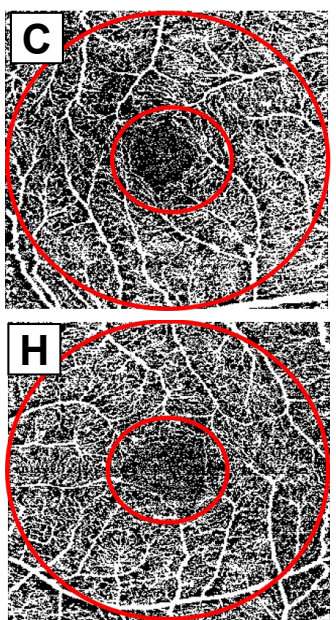

Whole retina
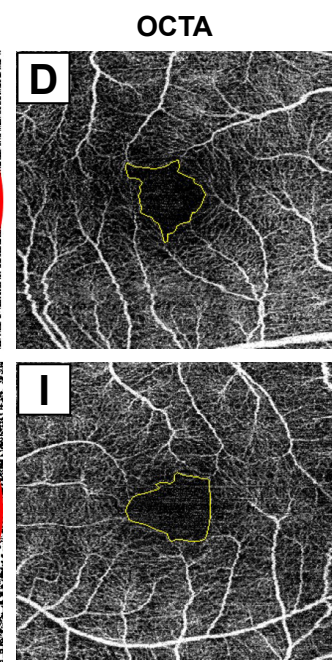

Binarization
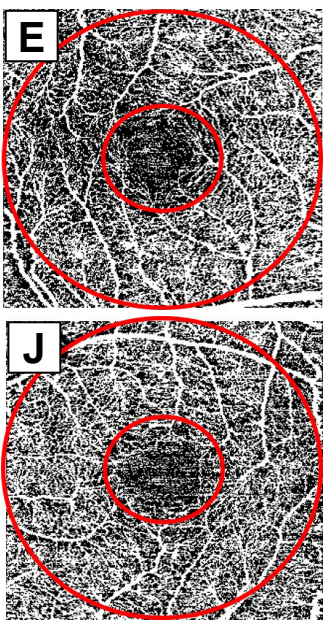

Figure 2 Measurements for representative eyes with macula-on RRD and fellow eyes.

Notes: Fundus photographs for the eye with macular-on RRD and the fellow eye (A, F). SS-OCTA en face images (B, D, G, I) and binarization images obtained with the Phansalkar method (C, E, H, J). The FAZ areas are measured for the superficial retina (B, G) and whole retina (D, I), and the vessel densities are measured using the binarization images and parafoveal areas (area enclosed by two concentric circles) for the superficial retina $(\mathbf{C}, \mathbf{H})$ and whole retina $(\mathbf{E}, \mathbf{J})$.

Abbreviations: RRD, rhegmatogenous retinal detachment; SS-OCTA, swept-source optical coherence tomography angiography; FAZ, foveal avascular zone. 
of the identifiable capillary network in the parafoveal area and the calculated area $\left(\mathrm{mm}^{2}\right)$ using ImageJ software (Figures 1 and 2).

\section{Statistical analysis}

All VD values and the FAZ area are expressed as medians (25th, 75th percentiles). The Wilcoxon-signed rank test was used to compare the axial length, VD, and FAZ area between the eyes with RRD and the fellow eyes. A $P$-value of $<0.05$ was considered statistically significant. All statistical analyses were performed using JMP version 10.1 software (SAS Institute Inc., Cary, NC, USA).

\section{Results}

Seven eyes of seven patients with macula-on RRD were initially recruited, and five of the eyes were eligible for analysis after application of the exclusion and inclusion criteria. Two eyes were excluded because of poor image quality for one eye and the presence of RRD in the parafoveal region for the other. The OCTA images used for analysis in all cases are shown in Figure S1. The patient's clinical characteristics were as follows: age, 49.0 years $(21.0,54.0)$; preoperative best-corrected visual acuity, $-0.08(-0.08,0.11)$ for RRD and $-0.08(-0.08,-0.03)$ for the fellow eye $(P=0.50)$; and axial length, $27.0(25.1,28.7) \mathrm{mm}$ for RRD and 27.4 (25.6, 28.5) $\mathrm{mm}$ for the fellow eye ( $P=0.31$; Tables 1 and 2$)$. The parafoveal VD $(P=1.00$ for the superficial retina and $P=0.44$ for the whole retina; Table 3 and Figure 3 ) and FAZ area $(P=0.31$ for the superficial retina and $P=0.13$ for the whole retina; Table 3 ) also showed no statistically significant differences between the eyes with RRD and the fellow eyes. Postoperative parafoveal VD values for the superficial and whole retina were available only for Cases 1 , 2 , and 4 (Figure S2), and there appeared to be no significant difference between the affected and fellow eyes.

\section{Discussion}

In this study, we demonstrated that the parafoveal VD and FAZ area remained unchanged in eyes with macula-on RRD.

Several studies have shown a relationship between RRD and macular flow reduction. Our previous study observed macular flow reduction in eyes with macula-on RRD using scanning laser Doppler flowmetry (SLDF). ${ }^{15}$ KubickaTrzaska and Górniak-Bednarz ${ }^{16}$ also reported that the blood flow in eyes with macula-on RRD was lower than that in the healthy fellow eyes using Doppler laser scanning. Because the methods and indices used in those studies were different from those used in our study, we could not perform direct comparisons. Nevertheless, extrapolation of individual results obtained with SLDF and OCTA can further our understanding regarding the interpretation of the findings of OCTA, which is a relatively new modality.

First, the measured parameters were different. SLDF used in the previous study evaluated the flow and velocity, ${ }^{17}$ whereas OCTA measured the VD, not the flow and velocity, in the present study. This helped in the detection of microvascular dropout, which was represented by a decrease in the vessel signal. ${ }^{12}$ Previous reports showed a relationship between RRD and endothelin-1 (ET-1), which is a powerful vasoconstrictor peptide, and increased levels of ET-1 in the plasma and subretinal fluid in patients with RRD. ${ }^{18}$ Our previous study reported that the macular vascular flow recovered after surgery for $\mathrm{RRD}$, and it was suggested that the macular flow in eyes with macula-on RRD may undergo a reversible change. ${ }^{15}$ Therefore, although SLDF can detect temporal changes in the velocity as a result of vasoconstriction, OCTA can detect the vascular structural changes that may be detected as capillary dropout. Moreover, SS-OCTA cannot detect reductions in the VD unless ischemia or severe flow reductions that lead to capillary dropout exist. Even if the vascular flow was reduced in the eyes with RRD in the present study, it may not have been severe enough to cause

Table I Characteristics of patients with macula-on RRD

\begin{tabular}{|c|c|c|c|}
\hline & RRD & Fellow eye & $P$-value ${ }^{a}$ \\
\hline Age (years) & \multicolumn{2}{|c|}{$49.0(21.0,54.0)$} & \\
\hline Sex (F/M) & \multicolumn{2}{|c|}{$0 / 5$} & \\
\hline Preoperative BCVA (logMAR) & $-0.08(-0.08,0 . \mathrm{II})$ & $-0.08(-0.08,-0.03)$ & 0.50 \\
\hline $\mathrm{IOP}(\mathrm{mmHg})$ & $13.7(|1.7| 5.7)$, & $13.0(\mid 1.8,16.5)$ & 0.88 \\
\hline SE (diopter) & $-6.25(-8.88,-2.95)$ & $-7.00(-9.50,-2.25)$ & 0.75 \\
\hline Axial length $(\mathrm{mm})$ & $27.0(25.1,28.7)$ & $27.4(25.6,28.5)$ & 0.31 \\
\hline
\end{tabular}

Notes: Data are presented as median (25th, 75th percentile). ${ }^{2}$ Wilcoxon signed rank test.

Abbreviations: RRD, rhegmatogenous retinal detachment; BCVA, best-corrected visual acuity; IOP, intraocular pressure; SE, spherical equivalent; F, female; M, male; logMAR, logarithm of minimum angle of resolution. 


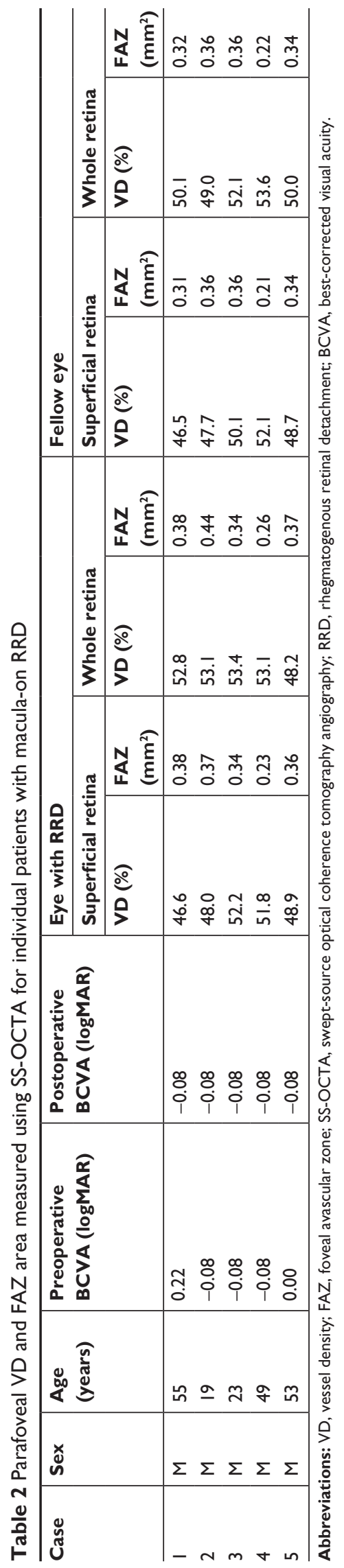

structural changes in the macular area. In fact, all patients in this study showed good pre- and postoperative visual acuities, this result supports the aforementioned hypothesis.

A second issue is the difference in the analyzed region. The measuring depth with SLDF was $300 \mu \mathrm{m}$, which can provide choroidal information, whereas the measuring area was superior and inferior to $10^{\circ} \times 2.5^{\circ}$ areas around the nasal macula. This considerably differs from the depth and area measured by OCTA in the present study. This may also explain the discrepancy in findings between the two studies, at least in part.

This study has several limitations that warrant discussion. First, the sample size was small, and quantitative analysis was possible for five and three cases before and after surgery, respectively. Although our limited data suggested that VD is not likely to show significant changes even after surgery, further studies with larger sample sizes are needed to confirm our findings. The second limitation is the presence of selection bias. We excluded subjects who could not undergo OCTA because of vitreous hemorrhage. A previous study reported that patients with RRD and vitreous hemorrhage had worse postoperative visual outcomes. ${ }^{19}$ Thus, we may have only included subjects with better macular function.Third, our study only included eyes with macula-on RRD. It would be interesting to include eyes with macular involvement (macula-off RRD) and perform comparisons between the two types. However, it is technically very difficult to obtain clear images for quantitative analysis, and our primary aim was to clarify the macular microvasculature analyzed using OCTA for eyes with macula-on RRD. Therefore, we only included eyes with a completely intact macula-on RRD diagnosed with fundoscopy as well as SD-OCT. Further studies should evaluate macula-off RRD eyes with only a shallow lift of the fovea or a detachment that is directly parafoveal. Despite these limitations, we were able to illustrate an intact microvasculature in eyes with macula-on RRD using a new modality that assesses the macular VD and FAZ area.

\section{Conclusion}

In conclusion, the findings of the current study suggest that the macular microvasculature is preserved in eyes with macula-on RRD. Macular microvasculature analysis using SS-OCTA has the potential to become a clinically relevant method for noninvasive and quantitative evaluations and is expected to provide useful information for understanding and monitoring the pathological macular status, if present, in eyes with RRD. 
Table 3 Parafoveal VD and FAZ area measured using SS-OCTA for eyes with macula-on RRD and fellow eyes

\begin{tabular}{l|l|l|l|l|l|l}
\hline \multirow{2}{*}{$\begin{array}{l}\text { OCTA } \\
\text { Segmentation }\end{array}$} & \multicolumn{2}{|l|}{ Parafoveal VD (\%) } & \multicolumn{2}{l}{ FAZ area $\left(\mathbf{m m}^{2}\right)$} \\
\cline { 2 - 7 } & RRD & Fellow eye & P-value $^{\mathrm{a}}$ & RRD & Fellow eye & -value $^{\mathbf{a}}$ \\
\hline Superficial retina & $48.9(47.3,52.0)$ & $48.7(47.1,51.1)$ & 1.00 & $0.36(0.28,0.37)$ & $0.34(0.26,0.36)$ & 0.31 \\
Whole retina & $53.1(50.5,53.2)$ & $50.1(49.5,52.8)$ & 0.44 & $0.37(0.30,0.41)$ & $0.34(0.27,0.36)$ & 0.13 \\
\hline
\end{tabular}

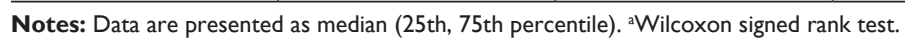

Abbreviations: VD, vessel density; FAZ, foveal avascular zone; SS-OCTA, swept-source optical coherence tomography angiography; RRD, rhegmatogenous retinal detachment.

\section{Superficial retina}

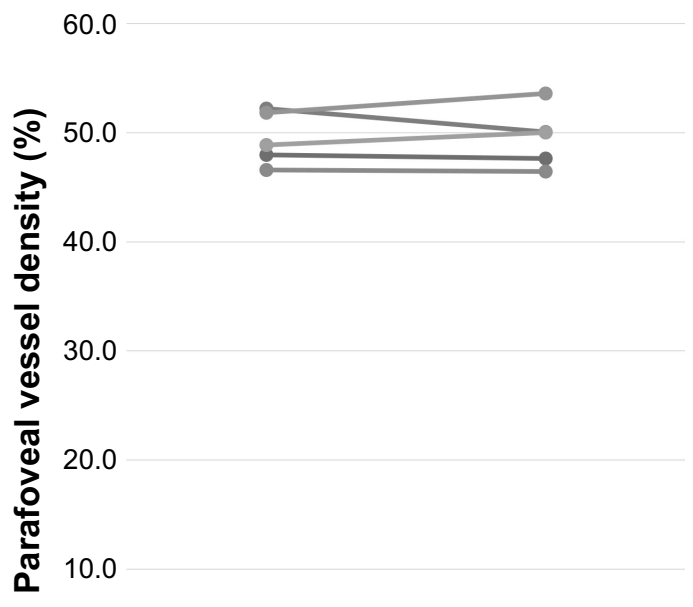

0.0

RRD

Fellow eye

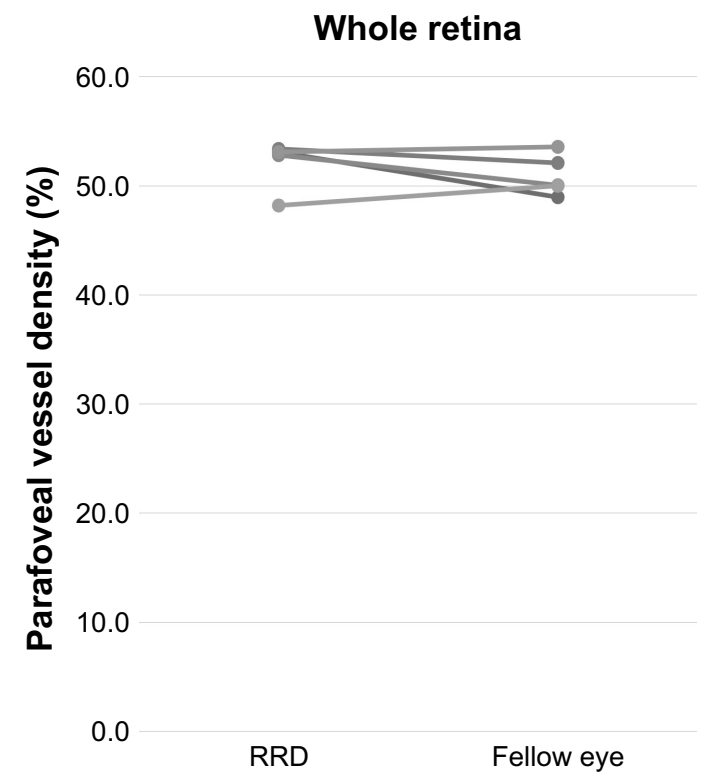

Figure 3 Plots showing the parafoveal VD values for eyes with macula-on RRD and fellow eyes.

Notes: The parafoveal VD in the superficial and whole retina is similar for the eyes with RRD and the fellow eyes. The coefficient of variation (mean [95\% CI]) for the values from the affected eyes and fellow eyes is $1.08 \%(0.03 \%-2.13 \%)$ for the superficial retinal layer and $2.04 \%(0.34 \%-3.74 \%)$ for the whole retinal layer.

Abbreviations: VD, vessel density; RRD, rhegmatogenous retinal detachment.

\section{Acknowledgments}

The authors thank Editage (https://www.editage.jp) for the English language review. This study was supported, in part, by the Grant-in-Aid for Young Researchers from Saitama Medical University Hospital and the Japan Society for the Promotion of Science (JSPS; KAKENHI grant numbers: 17K11430).

\section{Author contribution}

All authors contributed to data analysis, drafting and revising the article, gave final approval of the version to be published, and agree to be accountable for all aspects of the work.

\section{Disclosure}

The authors report no conflicts of interest in this work.

\section{References}

1. La Heij EC, Hendrikse F. Retinal detachments and retinal surgery. Ned Tijdschr Geneeskd. 1999;143(15):781-785.
2. Vaziri K, Schwartz SG, Kishor KS, Flynn HW. Tamponade in the surgical management of retinal detachment. Clin Ophthalmol. 2016;10: 471-476.

3. Ah-Fat FG, Sharma MC, Majid MA, Mcgalliard JN, Wong D. Trends in vitreoretinal surgery at a tertiary referral centre: 1987 to 1996. Br J Ophthalmol. 1999;83(4):396-398.

4. La Heij EC, Derhaag PF, Hendrikse F. Results of scleral buckling operations in primary rhegmatogenous retinal detachment. Doc Ophthalmol. 2000;100(1):17-25.

5. Roohipoor R, Mohammadi N, Ghassemi F, et al. Foveal structure in macula-off rhegmatogenous retinal detachment after scleral buckling or vitrectomy. J Ophthalmic Vis Res. 2015;10(2):172-177.

6. Maruko I, Iida T, Sekiryu T, Saito M. Morphologic changes in the outer layer of the detached retina in rhegmatogenous retinal detachment and central serous chorioretinopathy. Am J Ophthalmol. 2009;147(3): 489-494.

7. Baba T, Mizuno S, Tatsumi T, et al. Outer retinal thickness and retinal sensitivity in macula-off rhegmatogenous retinal detachment after successful reattachment. Eur J Ophthalmol. 2012;22(6):1032-1038.

8. Delolme MP, Dugas B, Nicot F, Muselier A, Bron AM, Creuzot-Garcher C. Anatomical and functional macular changes after rhegmatogenous retinal detachment with macula off. Am J Ophthalmol. 2012;153(1): 128-136.

9. Ito Y, Sasoh M, Ido M, Osawa S, Wakitani Y, Uji Y. Effects of scleral buckling without encircling procedures on retrobulbar hemodynamics as measured by color Doppler imaging. Arch Ophthalmol. 2005;123(7):950-953. 
10. Iwase T, Kobayashi M, Yamamoto K, Yanagida K, Ra E, Terasaki H. Changes in blood flow on optic nerve head after vitrectomy for rhegmatogenous retinal detachment. Invest Ophthalmol Vis Sci. 2016;57(14): 6223-6233.

11. Spaide RF, Klancnik JM, Cooney MJ. Retinal vascular layers imaged by fluorescein angiography and optical coherence tomography angiography. JAMA Ophthalmol. 2015;133(1):45-50.

12. Hwang TS, Jia Y, Gao SS, et al. Optical coherence tomography angiography features of diabetic retinopathy. Retina. 2015;35(11):2371-2376.

13. Spaide RF. Choriocapillaris flow features follow a power law distribution: implications for characterization and mechanisms of disease progression. Am J Ophthalmol. 2016;170:58-67.

14. Uji A, Balasubramanian S, Lei J, Baghdasaryan E, Al-Sheikh M, Sadda SR. Choriocapillaris imaging using multiple en face optical coherence tomography angiography image averaging. JAMA Ophthalmol. 2017;135(11):1197-1204.

15. Eshita T, Shinoda K, Kimura I, et al. Retinal blood flow in the macular area before and after scleral buckling procedures for rhegmatogenous retinal detachment without macular involvement. Jpn J Ophthalmol. 2004;48(4):358-363.
16. Kubicka-Trzaska A, Górniak-Bednarz A. Macular microcirculation blood flow changes after conventional surgery for rhegmatogenous retinal detachment. Klin Oczna. 2007;109(4-6):179-182.

17. Michelson G, Schmauss B, Langhans MJ, Harazny J, Groh MJ, Principle GMJ. Principle, validity, and reliability of scanning laser Doppler flowmetry. J Glaucoma. 1996;5(2):99-105.

18. Roldán-Pallarés M, Musa AS, Hernández-Montero J, Bravo-Llatas Sc C, Fernández-Durango R. Retinal detachment and proliferative vitreoretinopathy: central retinal artery blood velocities, intraocular pressure, and endothelin 1. Retina. 2013;33(8):1528-1539.

19. Yeung L, Yang KJ, Chen TL, et al. Association between severity of vitreous haemorrhage and visual outcome in primary rhegmatogenous retinal detachment. Acta Ophthalmol. 2008;86(2):165-169. 


\section{Supplementary materials}

\section{Before surgery}
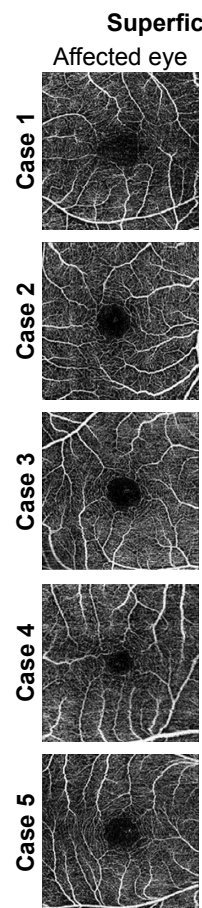
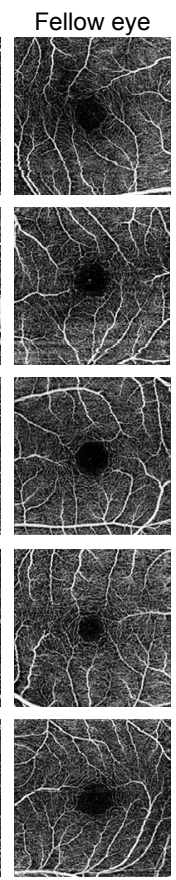

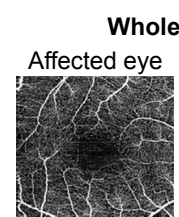

Whole retina
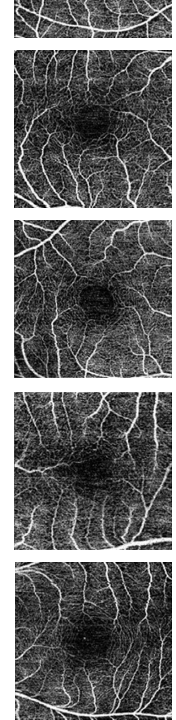

After surgery
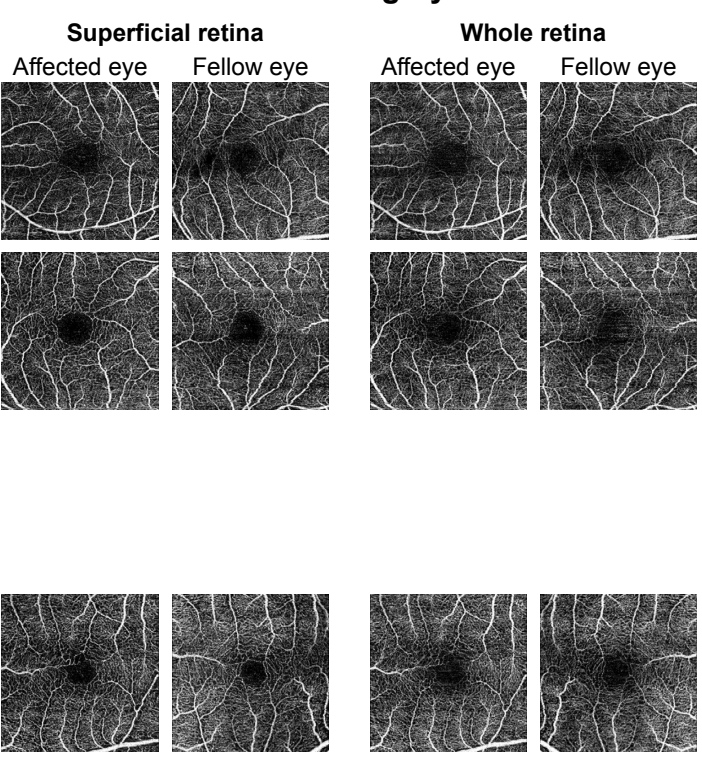

Figure SI OCTA images for five cases of macula-on RRD.

Notes: The raw OCTA images were used for quantitative analysis. The postoperative images for Cases 3 and 5 are not available. Abbreviations: OCTA, optical coherence tomography angiography; RRD, rhegmatogenous retinal detachment.

60.0

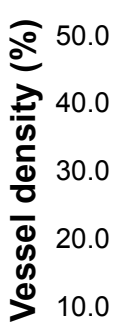

0.0

Pre-operative VD of SRL Post-operative VD of SRL (affected eye)

(affected eye)

60.0

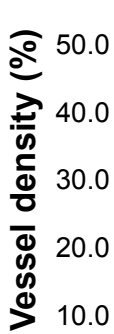

0.0

Pre-operative VD of WRL Post-operative VD of WRL (affected eye) (affected eye)
60.0

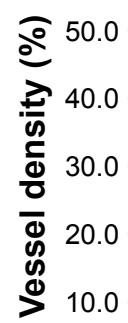

0.0

Pre-operative VD of SRL Post-operative VD of SRL (fellow eye) (fellow eye)

60.0

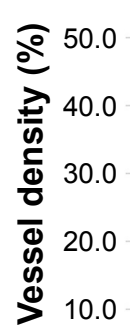

0.0

Pre-operative VD of WRL Post-operative VD of WRL (fellow eye) (fellow eye)

Figure S2 Plots showing the parafoveal VD values before and after surgery for eyes with macula-on RRD and fellow eyes.

Notes: The parafoveal VD in the superficial and whole retina is similar for the eyes with RRD and the fellow eyes. The coefficient of variation (mean [95\% CI]) for the values from the affected eyes and fellow eyes is $3.27 \%(-3.68 \%$ to $10.2 \%)$ for the superficial retina and $2.57 \%(-3.60 \%$ to $8.76 \%)$ for the whole retina. Abbreviations: VD, vessel density; SRL, superficial retinal layer; WRL, whole retinal layer; RRD, rhegmatogenous retinal detachment. 
Clinical Ophthalmology

\section{Publish your work in this journal}

Clinical Ophthalmology is an international, peer-reviewed journal covering all subspecialties within ophthalmology. Key topics include: Optometry; Visual science; Pharmacology and drug therapy in eye diseases; Basic Sciences; Primary and Secondary eye care; Patien Safety and Quality of Care Improvements. This journal is indexed on

Submit your manuscript here: http://www.dovepress.com/clinical-ophthalmology-journal

PubMed Central and CAS, and is the official journal of The Society of Clinical Ophthalmology (SCO). The manuscript management system is completely online and includes a very quick and fair peer-review system, which is all easy to use. Visit http://www.dovepress.com/ testimonials.php to read real quotes from published authors. 